九州大学学術情報リポジトリ

Kyushu University Institutional Repository

\title{
Note on the Estimation of the Mean Value of the Stochastic Process
}

Kudo, Akio

Kyushu University

https://doi.org/10.5109/12962

出版情報 : 統計数理研究. 5 (3/4)，pp. 53-58，1953-06. Research Association of Statistical Sciences バージョン :

権利関係 : 


\title{
NOTE ON THE ESTIMATION OF THE MEAN VALUE OF THE STOCHASTIC PROCESS
}

\author{
By Akio Kudô
}

\section{$\S 1$. Introduction.}

The main purpose of this paper is to discuss some combination of the theory of the linear estimation of the mean value function of the stochastic process due to U. GRENENDER [1] and the notion of the random integration of type A introduced by T. KITAGAwA [1]. In $\S 2$ we shall discuss the estimation of the mean value fnnction of the stochastic process under the condition that the continuous observation of the realization of the process in our concern is not available. $\$ 3$ is devoted to the discussion of the special case when the process is assumed to be stationary, and in this section we shall give certain estimations of the mean value which is assumed to be constant, and this hype of estimation has some connection with the computor of the integration such as recently planned in Japan. (IZAKI [1])

In preparation of this paper the author is deeply indebt to the valuable criterions and the warmest encouragement given by ProF. T. KitAGAwA.

\section{$\$ 2$. Combination with the notion of the random integration of type a.}

In his eminent paper, U. GRENANDER [1] discussed the linear estimation of the mean value of the stochastic process. The estimate of the mean value given by him is represented in the form of an integration of the realization of the stochastic process in our concern by certain function of bounded variation, i.e. in the following form

$$
m^{*}=\int_{o}^{T} x(t) d F(t),
$$

where $x(t)$ is a realization of the process in our concern. And the necessary and sufficient condition to $F(t)$ that $m^{*}$ should be the unbiased estimate of minimum variance is given by him. In his argument, the mean value function of the process is assumed to be constant. But his result can be obviously generalized to a fairly general case when the mean value function is a linear form of known functions.

And hence in this section without essential loss of generality we may and we shall assume that the mean value function of the process in our consern be constant and its variance fumction is 1 . On the other hand, he assumes that a continuous observation of the realization of the process is available to the statisticians. However, it may not always be possible, and even if it be possible, the numerical value of this integration may be perhaps neither 
directly nor strictly calculatable. Hence, we should adopt either of the following two methods, one of which is to aporoxlmate the vlaue of the integration with a finite RIEMANN sum, while the other is to estimate it by making use of the method of random integration of type $A$ introduced by $T$. Kitagawa [1]. And hence, some modifications to the formulations of the theory of statistical inference about stochastic process due to GRENANDER [1] seems to the author to be necessary, at least in view of its applications.

In this paper, we shall not here concern ourselves with the approximation with finite RIEMANN sum, because in this case we may consider the problem as the linear estimation for the process with an integral valued parameter.

Concerning the method of random integration of type $A$ we shall enunciate the following

Theorem 2.1. Let $x(t)$ be a stochastic process with unknown constant mean value $m=E(x(t))$ and with correlation function

$$
r(s, t)=E(x(s)-m)(x(t)-m),
$$

which is known to us. Let us assume that any realization of our process can be observed at any number of any time points over the time interval $(O, T)$ and let us draw $N$ time points of observation with the help of some chance mechanism in such a manner that these time points will be distributed over the interval $(O, T)$ mutually independently in the same distribution function $F(t)$. Here let us define an estimation of the mean value of our process as follows,

$$
m^{*}=\frac{1}{N} \sum_{i=1}^{N} x\left(t_{i}\right)
$$

Then it is an unbiased estimation and its variance is given by

$$
D^{2}\left(m^{*}\right)=\left(1-\frac{1}{N}\right) \int_{0}^{T} \int_{0}^{r} r(s, t) d F(t) d F(s)+\frac{1}{N} \text {. }
$$

Especially when we can choose the distribution function $F(t)$ satisfying the relation

$$
\int_{0}^{T} r(s, t) d F(t)=\text { constant }
$$

then the variance of this estimate is asymptotically equal to the variance of the unbiased estimate of minimum variance obtained by the conctiucns observation of the realization as $N$ becomes infinite, and this convergence being of the order $N$.

Proof. In view of the independency of $\left\{t_{i}\right\}$ we have

$$
D^{2}\left(m^{*}\right)=\frac{1}{N^{2}} \int_{o}^{T} \cdots \int_{o}^{T} \sum_{i, j=1}^{N} r\left(t_{i}, t_{j}\right) d F\left(t_{l}\right) \cdots d F\left(t_{N}\right)
$$




$$
\begin{aligned}
= & \frac{1}{N^{2}}\left\{2 \sum_{\substack{i, j=1 \\
i>j}}^{N} \int_{o}^{T} \int_{o}^{T} r\left(t_{i} t_{j}\right) d F\left(t_{i}\right) d F\left(t_{j}\right)+N\right\} \\
= & \int_{o}^{T} \int_{o}^{T} r(s, t) d F(s) d F(t) \\
& \quad+\frac{1}{N}\left\{1-\int_{o}^{T} \int_{o}^{T} r(s, t) d F(t) d F(s)\right\} .
\end{aligned}
$$

\section{$\S 3$. Discussion on the stationary case.}

GRENANDER proved in his paper [1] $\S 5$, when the process is assumed to have the constant mean value and be stationary, i.e. its correlation function is of the form

$$
r(s, t)=r(s-t)=\int_{-\infty}^{+\infty} e^{i \lambda(s-t)} d G(\lambda)
$$

and is purely non-deterministic, then under certain conditions the equidistributed estimate defined as follows

$$
\mu_{T}=\frac{1}{T} \int_{0}^{T} x(t) d t
$$

is an unbiased and asymptotically efficient estimate in the class of linear estimates, and hare let us denote its variance by $D^{2}\left(\mu_{T}\right)$, then we have the assertion

$$
D^{2}\left(\mu_{T}\right) \sim \frac{\pi}{T} G^{\prime}(0)
$$

where $G^{\prime}(0)$ is the spectral density at the origin of the stochastic process in our concern.

On the other hand, let us now take $N$ time points of observation $t_{1}, t_{2}$, $\cdots, t_{N}$, which are uniformly and mutually independently distributed over the time interval $(O, T)$ and let us define an unbiased estimate as follows

$$
\bar{\ell}_{T, N}=\frac{1}{N} \sum_{i=1}^{N} x\left(t_{i}\right) \text {. }
$$

Concerning the relation between the equi-distributed estimate and the estimate above defined, let us now ennunciate the following

Theorem 3. 1. Between $D^{2}\left(\mu_{T_{1}}\right)$ and $D^{2}\left(\bar{\mu}_{\psi_{2}, N}\right)$, which are the variance of $\mu_{T_{1}}$ and $\bar{\mu}_{T_{2}, v}$ respectively, there exists an asymptotical relation

$$
\frac{D^{2}\left(\mu_{T_{1}}\right)}{D^{2}\left(\bar{\mu}_{T_{2} . N}\right)} \sim \frac{\pi G^{\prime}(0)}{\pi G^{\prime}(0)\left(1-\frac{1}{N}\right)+\frac{T^{2}}{N^{2}} .}
$$


Here let us now consider the following estimations, which have scme connetions with the computor of the integration recently being planned in Japan. (see IzAKI [1]).

First let us draw time points $t_{1}, t_{2}, t_{3}, \cdots$ sequentially in such a way as they may be regarded as a PoIsson process i.e. the probability law of $t_{k}$ when $t_{k-1}$ is assigned is given in the following formulae;

$$
P_{r}\left(t_{k} \leq t_{k} \leq t_{k}+d t_{k} / t_{k-1}\right)=a e^{-a\left(t_{k}-t_{k}-1\right)} d t_{k} .
$$

Further let us define a random variable $M$ by the following relation

$$
t_{N-1} \leq T \leq t_{M} .
$$

Here we note that $a$ is the average number of the time points in a unit interval.

Here let us define two estimations of the mean value of our stochastic process as follows;

First let us define the following estimation $m^{*}$,

$$
m^{*}=\frac{1}{M-1} \sum_{i=1}^{M-1} x\left(t_{i}\right),
$$

where $M$ is defined as follows: First we take series of time points of observations in the above mentioned manner and if $M$ defined by (3.7) is not equal to one we take this number as $M$ of (3.8), and on the other hand if $M=1$ we repeat the drawing of the time points until it becomes $M \neq 1$, and take this number as $M$ of (3.8).

Another estimation of the mean value of our process is defined as follows

$$
m^{* *}=\frac{1}{T} \sum_{k=1}^{M}\left(t_{k}-t_{k-1}\right) x\left(\xi_{k}\right),
$$

where $M$ is a number defined by (3.7) and $t_{0}=0, t_{M}=T$ and $\xi_{k}(k=1,2$, $\cdots, M$ ) are time points of observations uniformly and mutually independently distributed over the interval $\left(t_{k}, t_{k-1}\right)$ respectively.

Here let us remark that this estimation of the mean value may be obtained when we estimate it by making use of the computor of the integration above mentioned.

Concerning these two estimations we have following

Theorem 3.2.

$$
\begin{aligned}
E\left(m^{*}\right) & =E\left(m^{* *}\right)=m \\
D^{2}\left(m^{*}\right) & =D^{2}\left(\mu_{T}\right)+\frac{1-D^{2}\left(\mu_{T}\right)}{e^{a T}-1} \sum_{N=1}^{\infty} \frac{(a T)^{v}}{N ! N}=D^{2}\left(\prime_{T}\right)+O\left(\frac{1}{a T}\right) \\
D^{2}\left(m^{* *}\right) & =D^{2}\left(\mu_{T}\right)+O\left(\frac{1}{a T}\right),
\end{aligned}
$$

where we set

$$
D^{2}\left(\mu_{T}\right)=\frac{1}{T^{2}} \int_{0}^{T} \int_{0}^{T} r(s, t) d s d t
$$


Especially when our process is assumed to be stationary and the correlation function $r(t)$ is analytic in $t>0$ and expressible in the following form,

$$
r(t)=1+c t^{\alpha}+\sum_{k=\alpha+1}^{\infty} c_{k} t^{k}, \text { for } t>0
$$

where $\alpha$ is some positive integer.

Then the variance is given in the following

$$
D^{2}\left(m^{* *}\right)=D\left(\mu_{T}\right)-2 \ltimes ! c e^{-a T} T^{\alpha} \sum_{N=1}^{\infty} \frac{N(a T)^{N-1}}{(\alpha+N+1) !}+O\left(\frac{1}{a^{\alpha+2} T}\right)
$$

and

$$
e^{-a T} T^{\alpha} \sum_{N=1}^{\infty} \frac{N(a T)^{N-1}}{(\alpha+N+1) !}=O\left(\frac{1}{a^{\alpha+1} T}\right) .
$$

Proof. The unbiasedness of $m^{*}$ and $m^{* *}$ is clear and we shall here give the calculation of the variance of $m^{* *}$. The calculation of the variance of $m^{*}$ can be done in the same way as that of $m^{* *}$.

The conditional variance of $m^{*}$ when $\left\{t_{k}\right\}$ and $\left\{\xi_{k}\right\}$ are assigned is given in the following

$$
\frac{1}{T^{2}} \sum_{k, l=1}^{N}\left(t_{k}-t_{k-1}\right)\left(t_{l}-t_{l-1}\right) r\left(\xi_{k} \xi_{l}\right)
$$

and in view of the conditions to $\left\{\boldsymbol{t}_{k}\right\}$ and $\left\{\boldsymbol{\xi}_{k}\right\}$ we are lead to the calculation of the variance as follows,

$$
\begin{aligned}
& T^{2} D^{2}\left(m^{* *}\right)=\sum_{A=1}^{\infty} \int_{0 \leq t_{1} \leq t_{2} \leq \cdots \leq t_{N^{*}-1} \leq T \leq t^{\prime} N^{\prime}<\infty} \int \\
& \left\{\sum_{k, l=1}^{N}\left(t_{k}-t_{k-1}\right)\left(t_{l}-t_{l-1}\right) \boldsymbol{r}\left(\xi_{k} \xi_{l}\right)\right\} \\
& \left\{\prod_{k=1}^{N} \int_{t_{k-1}}^{t_{k}} \frac{d \hat{\Sigma}_{k}}{t_{k}-t_{k-1}}\right\}\left\{\prod_{k=1}^{N-1} a e^{-a\left(t_{k}-t_{k}-1\right)} d t_{k}\right\} a e^{-a\left(t_{N^{\prime}}-t_{N^{-1}}\right)} d t_{N}^{\prime} \\
& =e^{-a T} \sum_{N=1}^{\infty} a^{N-1} \int_{0 \leq t_{1} \leq t_{2} \leq \cdots \leq t_{N-1} \leq T} \int \\
& \left\{2 \sum_{\substack{k, l=1 \\
k>l}}^{N} \int_{t_{k-1}}^{t_{k}} \int_{t_{l-1}}^{t_{l}} r(s, t) d s d t+\sum_{k=1}^{N}\left(t_{k}-t_{k-1}\right)^{2}\right\} \\
& d t_{1} d t_{2} \quad d t_{N-1} \\
& =e^{-a T} \sum_{N=1}^{\infty} a^{N-1} \frac{T^{N-1}}{(N-1) !} \int_{0}^{T} \int_{0}^{T} r(s, t) d s d t \\
& +e^{-a T} \sum_{N=1}^{\infty} a^{N-1} \int_{0 \leq t_{1} \leq t_{2} \leq \cdots \leq t_{N-1} \leq T} \int \\
& \left\{\sum_{k=1}^{N} \int_{t_{k-1}}^{t_{k}} \int_{t_{k-1}}^{t_{k}}(1-r(s, t)) d s d t\right\} d t_{1} d t_{2} \cdots d t_{N-1} \\
& =\int_{0}^{T} \int_{0}^{T} r(s, t) d s d t+I
\end{aligned}
$$


On the other hand, we have

$$
\begin{gathered}
\int_{0 \leq t_{1} \leq t_{2} \leq \cdots \leq t_{N-1} \leq T} \int \sum_{k=1}^{N}\left(t_{k}-t_{k-1}\right)^{n} d t_{1} d t_{2} \cdots d t_{N-1} \\
=\frac{(n) ! N}{(n+N-1) !} T^{n+N-1} .
\end{gathered}
$$

Hence we have in vicw of the infquality $\mid 1-r(s, t) \leq 2$,

$$
\begin{aligned}
I & \leq 2 T^{2} e^{-a T} \sum_{N=1}^{\infty} \frac{(a T)^{N-1}}{(N+1) !}(2 N) \leq 4 T^{2} \sum_{N=1}^{\infty} \frac{(a T)^{N-1}}{N !} \\
& =4 \frac{T}{a} e^{-a T}\left(e^{a T}-1\right)=O\left(\frac{T}{a}\right) .
\end{aligned}
$$

Hence (3.15) is proved.

And on the other hand, if the condition (3.14) is fulfilled, we have

$$
\begin{aligned}
& \text { (3.20) } T^{2} D^{2}\left(m^{* *}\right)-\int_{0}^{T} \int_{0}^{T} r(s, t) d s d t \\
& \quad-2 e^{-a T} \sum_{N=1}^{\infty} a^{N-1} \frac{c}{(\alpha+1)(\alpha+2)} \int_{0 \leq t_{1} \leq t_{2} \leq \cdots \leq t_{N}-1 \leq T} \int_{k=1}^{N}\left(t_{k}-t_{k-1}\right)^{\alpha+2} d t_{1} \cdots d t_{N}, \\
& \quad+I_{2} \\
& =\int_{0}^{T} \int_{o}^{T} r(s, t) d s d t-2 c \alpha ! e^{-a T} \sum_{N=1}^{\infty} \frac{N}{(\alpha+N+1) !} a^{N-1} T^{\alpha+N+1} \\
& \quad+I_{2} \\
& =T^{2} D^{2}\left(\mu_{T}\right)+I_{1}+I_{2}
\end{aligned}
$$

and

$$
\left|I_{1}\right| \leq 2 c \mid \alpha ! e^{-\alpha T} a^{-(\alpha+1)} T \sum_{N=1}^{\infty} \frac{(a T)^{\alpha+N}}{(N+\alpha) !}=O\left(\frac{T}{a^{\alpha+}}\right)
$$

and hence

$$
I_{2}=O\left(\frac{T}{a^{\alpha+2}}\right)
$$

which is to be proved.

\section{References}

Grenander, $\mathrm{U} \cdot[1]$ : Stochastic process and statistical inference, Arkiv för Mathematik, Band 1 Hafte (1950), p. - p.

IzAKI, M. [1]: Convergence of integral and its applications. Bull. of Math. Stat. Vol. 5, No. $1-2(1952)$, p. $31-34$.

Kitagawa, T. [1]: Random integrations. Bull. of Math. Stat. Vol. 4, No. 1-2, (1952), 1-7.

$\mathrm{K}_{\mathrm{ITA}} \mathrm{AAW}, \mathrm{T}$. [2]: Sampling from the processes depending upon a continuous parameter, Men. Fac. Kyushu Univ., Ser. A, Vol. 5, No. 2 (1950), 181-188. 